\title{
Aging evaluation of high power lithium cells subjected to micro-cycles
}

\author{
M. Ceraolo ${ }^{1}$, G. Lutzemberger ${ }^{1}$, D. Poli ${ }^{1}$ \\ ${ }^{1}$ Department of Energy, Systems, Territory and Constructions Engineering, University of Pisa, \\ Largo Lucio Lazzarino n. 1, 56123, Pisa (Italy) \\ *Corresponding author: tel. +39050 2217311, \\ email: lutzemberger@dsea.unipi.it
}

\begin{abstract}
A typical operating condition of storage batteries requires to deliver and absorb small currents in large intervals of time, in order of minutes or hours. However, in the last years lithium batteries have been more and more considered in "power oriented" applications, in which they are required to manage large currents in short intervals of time, typically a few seconds or tens of seconds. Unfortunately, very limited information about this kind of usage is available in literature, in terms of battery performance and aging.
\end{abstract}

Therefore, the paper focuses firstly on the experimental evaluation of performance of high power and super high power lithium batteries also in comparison to other power oriented storage systems adequate for use onboard hybrid vehicles, such as supercapacitors (SCs). The evaluation has been performed through experimental tests. Results have shown that these batteries are able to guarantee significant performance, even higher than data declared by manufacturer, with slight over-temperature.

Then, for high power lithium batteries aging is discussed, when they are subjected to shallow-depth charge/discharge cycles. The aim is to evaluate if the battery life corresponding to such micro-cycles can reach several hundreds of thousands that are required for applications such as hybrid vehicles and hybrid stationary generation systems. Also in this case experimental tests able to prove it have been executed. They have shown a substantially unaltered capacity fade during the execution of hundreds of thousands of microcycles, thus confirming the vocation of these devices for power-oriented applications.

Keywords: Aging; Cycle life; High power cell, Hybrid vehicles; Lithium battery; Supercapacitor. 


\section{Introduction}

A frequent operating condition for storage batteries requires to deliver and absorb relatively small currents in large intervals of time, typically tens of minutes or hours. However, energy storage systems more and more are required to deliver or absorb very high currents in short intervals of time, typically a few seconds or tens of seconds. This kind of usage is commonly described saying that this energy storage is "power oriented", opposed to the conventional "energy oriented". As example, three main case studies can be cited.

- Hybrid vehicles. According to a standard definition (ISO/TR 8713), the power needed from propulsion comes from at least two independent sources at least one of which being reversible. Typically, one path is composed by a "fueled source", here called primary converter, the other one by an, electrochemical energy storage system. According to the most common energy management strategy, the primary converter delivers average load powers, while the battery delivers or absorbs the ripple around that average, thus delivering energy during acceleration, or recovering energy during braking. Typically, these acceleration and braking phases involve durations in the order of seconds or tens of seconds, and require relatively high currents. More precisely, considering that currents are usually given as multiple of the nominal capacity, we can estimate values higher than $10 C_{\mathrm{n}}[1],[2],[3],[4],[5]$

- Tramways. In the most common tramway systems, the feeding points (electrical substations) power flow cannot be reversed. Therefore, the braking energy of a tram can be recovered only in case there are other trains capable of absorbing that energy. In case, however, the feeding network is equipped in one or more points with an energy storage system, it can absorb energy during braking of some trams, and send it back to the traction system, whenever there is request from the load. This solution promises to enlarge the quantity of the recovered energy significantly. Therefore, the energy storage is subjected to high peak currents up to $10 C_{\mathrm{n}}$ when the tram is breaking or accelerating, typically for durations of around 10-20 s [6], [7].

- Stationary applications. In recent years, electricity grids moved towards an increased share of renewable energy sources (RES), which are continuously being connected to the system and have a 
distributed nature. RES's, however generate energy in an undispatchable way; sending large RES amount of energy into the grid might cause network stability problems as well as reduced efficiency of other power sources on the grid [8], and therefore it is often highly advisable to store this energy locally to deliver to loads at later times, i.e. when the local generation-load balance allows.

In some of the examined cases, the main concern of using lithium batteries at high currents is during charging, since manufacturers impose limits on the charging current that imply much smaller charging powers than discharging ones. An additional concern over the proposed usage of lithium batteries regards the cell life. In fact, manufacturers typically consider full-depth charge-discharge cycles at continuous, low currents (i.e. $0.5 \div 1 C_{\mathrm{n}}$ ) for which a life of few thousands of cycles is expected [9]. On the other hand, no data are often available for shallow-depth cycles at high currents, hereinafter called "micro-cycles", characterised by a SOC fluctuation lower than $5 \%$, and amplitudes at least higher than $5 C_{\mathrm{n}}$.

Also scientific literature lacks details on this topic. In fact, identification of the aging mechanism by analysis of equivalent electrical models, typically involve full-depth SOC fluctuation and reduced current rates [10], [11], [12]. In some cases, dependence by thermal effects has been considered [13]. Indeed, aging effects have been also evaluated by performing charging and discharging cycles at highest temperature than ambient [14].

Much more sophisticated techniques are currently in use. As example, a new model for expressing the differential capacity characteristics for $\mathrm{SOH}$ estimation, directly associated with the transition behavior of active materials is proposed in [15]. Additionally, also EIS (Electrochemical Impedance Spectroscopy) can be often considered [16]. The changing of the impedance spectra of the lithium ion cells can be analysed starting from the new cell, and evaluating the cycle or calendar life of the aged cell. Then, once disassembled, a post mortem analysis can be also performed [17] [18] [19]. However, also in this case the cell is aged through full depth charging-discharging cycles, executed at reduced current rates, far from the real cell usage in power oriented applications.

Only in a reduced number of cases, aging effects have been analysed considering high charging or discharging rates. Unfortunately, only full depth cycles have been considered [20], or asymmetric testing conditions have been selected, i.e. maintaining low charging currents in combination with high discharging 
current rates [21]. In some other few cases, shallow cycles have been directly investigated. However, DOD does not reach lower values than $20 \%$ [22], although sometimes currents higher than $5 C_{\mathrm{n}}$ are considered. In other cases, current rates remain significant lower [23].

Aging can be also analysed in reference with lithium cells engaged in electric vehicular applications. In this regard, plug-in hybrid and electric vehicles are mainly considered. However, they typically make use of expressively energy oriented battery pack, normally subjected to full charging-discharging profiles, executed at reduced current rates [24] [25] [26] [27]. It must finally be said that standard regulations [28] [29] used for laboratory testing activities are typically able to reproduce only in an idealized way the effective stress which the cell is effectively subjected. In fact they typically consider constant charging or discharging current phases. On the contrary much more realistic profiles, according to real stress which the battery is subjected, should be carefully considered, as will be discussed later.

In conclusion, all the presented capacity fade technique analysis are typically made in relation to fulldepth SOC fluctuation and reduced current rates, indeed referring to unrealistic stress conditions for power oriented applications. Starting from that, the paper focuses on the experimental evaluation of the aging process for high power lithium batteries subjected to high-current shallow cycles. A briefly evaluation of preliminary results on analogue stress characteristics was also shown in [30]. The aim is to evaluate whether the battery life corresponding to such micro-cycles can reach several hundreds of thousands that are required for applications such as hybrid vehicles and hybrid stationary generation systems.

In terms of the considered methodology, according to the theory that aging is thermally induced, it must be carefully assessed if shallow charging-discharging cycles, i.e. micro-cycles in which high current rates are maintained for a short time, may be responsible of slight over-temperature for the battery. If so, it can be assumed after experimental verification that under these stress conditions aging can effectively occur after several hundreds of thousands of micro-cycles.

A similar approach was followed also in [31] and verified on analogue testing conditions, reproducing the behaviour of hybrid vehicles. Some of the aging results achieved for the cell used in [31] have been here briefly recalled, with the main aim to give much more strength in terms of statistical validity to the obtained results. 


\section{Cell performance}

To verify the performance of power oriented lithium batteries, two devices have been selected from the market. The first one, for high power applications, the second one, for super-high power applications, in which higher limits for discharging pulse currents were indicated. The relative characteristics are described below. Firstly, super high power cells were compared with supercapacitors, another energy storage system typology expressively power oriented. After that, much more representative tests of effective usage in high power applications were performed also in case of high power cells, reducing durations for charging and discharging, and increasing currents. In particular they started from general stylized shapes, such as rectangular and linear shapes, coming up to representation for real existing stresses, such as current profiles derived from simulation of existing hybrid powertrains.

\subsection{Devices under test and laboratory setup}

The first device under test is one lithium cell capable of high power ${ }^{1}$, having a nominal, two-hour declared capacity of $13.0 \mathrm{Ah}$.

Performance data for this cell are available for full discharges to up to $8 C_{n}$, while pulse discharges may be performed up to $15 C_{n}$, where $C_{n}$ represents the nominal battery capacity, reported in manufacture's datasheet, expressed in Ah.

From manufacturer's graphical documentation reported in [9] the numerical data of Table 1 can be inferred, (where the discharge regime, as already mentioned, is reported in ampere per nominal Ah of battery capacity) that confirms the definite vocation of this battery for high powers, since very little charge penalty occurs.

According to this table therefore a single cell, whose mass is $332 \mathrm{~g}$, should be able to deliver, at $8 C_{\mathrm{n}}$ regime, around $1096 \mathrm{~W} / \mathrm{kg}$. However it is not clear how long this discharge regime can be maintained without battery damage, nor what are the current limits during charge, since indication on pulse charging is missing (i.e. only full charges up to $3 C_{n}$ are considered) and not, finally, how many times charge/discharge cycles at high currents can consecutively be applied.

\footnotetext{
${ }^{1}$ NMC Lithium cell of the "High Power" family, 13.0 Ah, from [9].
} 
The other device under test is one of the lithium cell capable of the highest power on the market ${ }^{2}$. Performance data for this battery are available for full discharges up to $15 C_{n}$ while pulse discharges may be performed up to $20 C_{n}$. According to manufacturer data, this cell whose mass is $226 \mathrm{~g}$ should be able to deliver at $15 C_{n}$ regime, around $1673 \mathrm{~W} / \mathrm{kg}$, significantly higher than the previous case. Main data and performance in terms of sustainable currents declared by the manufacturer for the two considered cells have been summarized in Table 2 .

No data about pulse charging is given, in terms of current amplitude or time duration. In this regard, a campaign of experimental tests is needed, to correctly characterise the battery performance in terms that are immediately usable for all the high power applications.

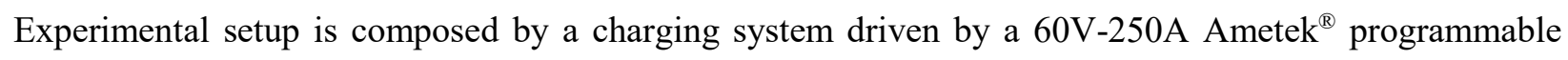
DC power supply (Model SPS60X250-K02D). Discharging system is instead a 60V-500A Zentro-Elektrik ${ }^{\circledR}$ Electronic Load (Model EL6000). The two systems can be fully remote controlled via GPIB standard interface, through a software developed in LabView ${ }^{\circledR}$ environment. About measurement system, current is measured using a shunt, class 0.5 and full scale $150 \mathrm{~A}$; the voltage is measured with a DAQ device (Model NI 9219) with a declared accuracy of $0.3 \%$ of reading. The device under test is finally located in a climatic chamber Binder ${ }^{\circledR} \mathrm{MK} 53$, characterized by a temperature range $-40^{\circ} \mathrm{C}$ to $180^{\circ} \mathrm{C}$. All the presented tests have been executed in the climate chamber fixed at $22^{\circ} \mathrm{C}$.

\subsection{SC comparison}

First of all, it is of interest to compare the most promised lithium technology taken into account, i.e. the super high power cell, with a $20 \mathrm{~F} 15 \mathrm{~V}$ supercapacitor module ${ }^{3}$, whose data are shown in [32]. In fact, supercapacitors is one of the most famous power oriented energy storage device, and its usage is typically considered in high power applications.

The main aim of the comparison was indeed to verify if the power delivered by the lithium battery in charging or discharging phases can compete with a device expressively power oriented. Naturally, cycle life of supercapacitors is typically indicated in around one million of charging-discharging cycles, thus always

\footnotetext{
${ }^{2}$ NMC Lithium cell of the "Ultra High Power" family, 7.2 Ah, from [9].

${ }^{3}$ Power type ultra-capacitor module 20 F $15 \mathrm{~V}$, from [33].
} 
higher than what expectable by a lithium battery, whose cycle life strictly depends, as discussed later, by the depth of discharge.

However, to make a fair comparison of different storage systems for hybrid vehicles, they must be subjected to the same stress; in particular, their specific power must be assessed using the same discharge duration. Therefore the tests used in the comparisons described in [32] are based on the discharge time that, coherently with the typical usage of a storage system onboard a vehicle, is between a few seconds and a few minutes. This is very important because figures often used such as "matched impedance" power for supercapacitors (that, as al-ready noted can be sustained only for a fraction of a second) or battery nominal power (often determined for discharges of around a minute) cannot be effectively compared to each other. In [32] stress types based on specific power test, constituted by a full I-U charge followed by full constant-I discharge at different regimes, and efficiency tests, constituted by repeated charge-discharge cycles, have been used.

When testing supercapacitors, discharge started at maximum capacitor voltage (i.e. from fully charged capacitor) and terminated when the terminal voltage was half the initial value; when testing batteries, discharge ended when the mini-mum voltage of $2.7 \mathrm{~V} /$ cell was reached, considering the lowest voltage cell, to avoid battery damage.

To evaluate specific powers, the charging current was set at the same value used for discharging for SCs (symmetric charging), while for battery it was held constantly to $3 C_{\mathrm{n}}$, the maximum allowed by the battery manufacturer, corresponding to a specific power of $330 \mathrm{~W} / \mathrm{kg}$ (for batteries I-U charge was used).

Results in terms of specific power vs specific energy are shown in Figure 1, where Ragone plots for the two considered devices have been presented.

As it is possible to observe, the competitiveness of super high power lithium batteries with respect to supercapacitors seems very high when only discharge is considered. If the usual $3 C_{n}$ limitation during charge is introduced, the competitiveness of Li-Bat is greatly reduced, but still evident starting from around $30 \mathrm{~s}$ time. Indeed, the competitiveness of super high power lithium batteries with respect to supercapacitors seems 
very high when only discharge is considered, while when vehicle stress considered implies discharge in the order of one minute, SCs alone cannot compete.

Then, temperature has been monitored for the super high power cell, to analyze stress under the highest discharging currents and durations, under the same charging conditions. Results are shown in Figure 2. They show that if discharging currents as high as $10 C_{\mathrm{n}}$ or $20 C_{\mathrm{n}}$ are used, battery temperature control can be a problem, mainly in case of long time durations (i.e. $500 \mathrm{~s}$ ), although at reduced current (i.e. $10 C_{\mathrm{n}}$ ).

However, the tests already described, although meaningful to compare the two devices, are not fully representative of realistic usage of these high power batteries, since imply long discharge durations. The problem to be addressed is indeed whether the analysed limits, particularly during charging operation, can be overcome on realistic usage, as detailed in the next section.

\subsection{Stress definition}

The stress to which the considered storage device is to be subjected should reproduce, in a schematic idealised way, the stress that are encountered in power oriented applications.

The executed shallow charging-discharging cycles have been performed testing the lithium cells in a climatic chamber, at a reference ambient temperature of $22^{\circ} \mathrm{C}$. In this regard, it may be questionable if thermal conditions referred to one single cell may be representative of those related to the whole battery pack. Naturally, they cannot fully reproduce the thermal phenomena due the mutual interaction between cells, neither the effects of cooling system installed on-board vehicle. On the other hand, it must be specified that starting with the analysis of the whole battery pack should be extremely complex, mainly in relation to the results to be interpreted. Thus, the attention has been posed at first stage on one single cell, leaving to further analysis the execution of aging tests on the complete battery pack.

The repeated shallow charge-discharge cycles, i.e. micro-cycles, reproduced during lab tests have been realised in three different ways:

A. Micro-cycles performed at constant current, symmetric in charging and discharging phases, without overcoming the battery over-temperature limit. These cycles imply shallow DOD, and are significant, in idealistic way, of battery operation in general "power-oriented" applications. This kind 
of test has been performed with the two available devices, i.e. high power and super-high power cells.

B. Micro-cycles performed at linearly variable current, symmetric in charging and discharging phases. These are much more significant of battery operation in hybrid vehicle applications. For this kind of test, the high power cell was used.

C. Variable micro-cycles, executed at constant or linear current, to reproduce in much more realistic way the stress that is subjected the battery of a series-hybrid vehicle, based on common battery management strategy, performing a low powered NEDC cycle [34]. This test was performed using the super high power cell.

One single partial cycle of type A (constant current) is shown in the left part of Figure 3: it is constituted by constant-charge, rest phase and constant-discharge, with the following constraints:

- Duration and current amplitude of charge $(a b)$ and discharge $(c d)$ phases are the same. Values are chosen to maintain the lithium cell within the admitted window voltage $(4.2-2.7 \mathrm{~V})$. Amplitudes have been chosen equal to $5 C_{\mathrm{n}}, 7.5 C_{\mathrm{n}}, 10 C_{\mathrm{n}}$, while corresponding durations are, respectively, equal to $30,20,10$ seconds.

- The rest phase $(b c)$ has a fixed duration of $20 \mathrm{~s}$.

For each current amplitude and duration, the battery temperature was measured along the test. This way, the maximum charge/discharge current compatible with limits of voltage and thermal conditions can be evaluated.

On the other hand, test of type B represents the current to which the battery is subjected during typical of hybrid vehicle usage, operating in standardised driving cycles [34]. In fact, these are characterised by constant acceleration and deceleration. Thus, also the wheel traction force stays, under the hypothesis to neglect the aerodynamic drag contribution (i.e. proportional to the square of velocity), almost constant, and the wheel power, consequently, varies linearly with speed. Additionally, in a series hybrid drive train of the type described in [1], [2], [3], [4], [5], apart losses, the power profile remains unchanged moving from mechanical wheels to the electric drive. Then, using a common energy management strategy [35], the 
primary converter delivers the average power requested for propulsion, while the battery delivers or absorbs the ripple around that average.

Indeed, the battery power, and then the battery current if considering almost constant voltage during charge-discharge micro-cycles, can be effectively idealised in a triangular profile at zero mean value, as represented in the right part of Figure 3, in which a single partial cycle of type B is shown. Similar constraints to the previous case have been considered:

- Duration and peak current amplitude of charge $(a b)$ and discharge $(c d)$ phase are the same. Amplitudes have been chosen equal to $10 C_{\mathrm{n}}$, while corresponding durations are, respectively, equal to $20 \mathrm{~s}$ and $10 \mathrm{~s}$.

- $\quad$ The rest phase $(b c)$ has a fixed duration of $20 \mathrm{~s}$.

For each current amplitude and duration, the battery temperature was measured along the test.

Finally, test reproducing a vehicle performing the NEDC cycles was introduced. It is well known that the NEDC cycle is composed by the four repetitions of the urban part, plus a sub-urban part [34]. The sub-urban part has normally a maximum speed of $120 \mathrm{~km} / \mathrm{h}$, but for low powered vehicles, a version limited to $90 \mathrm{~km} / \mathrm{h}$ may be used. The same drivetrain typology and energy management strategy considered in determination of type B stress have been used. The resulting current is shown in Figure 4, where the speed profile is shown as well; to make the plots clearer, only two urban parts of this cycle are displayed. As visible, coherently with the constant acceleration phases, triangular shapes for current profile are confirmed, although of different amplitudes in comparison to the idealized type B stress, due the complexity of the speed profile. Then, since the maximum battery stress occurs during the last vehicle braking action, it is seems reasonable to give up part of the energy that could be recovered during the last brake, limiting the maximum charge to a value that allows the battery to be sized with a symmetrical profile, i.e. with equal maximum charge and discharge current. As far as the actual maximum value of the peak currents, considering the results from type A test, a value of $6 C_{\mathrm{n}}$ has been chosen. 


\subsection{Basic results}

One sample test of type A related to an amplitude of $10 C_{\mathrm{n}}$ and duration of $10 \mathrm{~s}$ is shown in Figure 5. As visible, the difference between the cell temperature and the ambient becomes constant after few cycles, and reaches the maximum values of about $36^{\circ} \mathrm{C}$, in the considered scenario. The main limitation is due the maximum permissible lithium cell voltage, equal respectively to $4.2 \mathrm{~V}$ : when the cell is near the full charge condition, pulse charges must be interrupted, as visible in the first part of Figure 5, not to overcome the maximum allowable voltage. The opposite occurs when it is near to the full discharging condition. In this regard, the cell finds automatically the right SOC to not overcame the maximum and minimum voltage limits, because cuts in charge or discharge currents make the cycle unbalanced.

Actually, this aspect is emphasized when the cell temperature is still close to the ambient temperature, since the cell overheating causes a significant reduction, as visible always from Figure 5, of the operating window voltage.

The global set of results for the high power cell is summarised in Table 3, where are reported the maximum cell over-temperature $\Delta \mathrm{T}$ from ambient at $22^{\circ} \mathrm{C}$, and the maximum $\Delta \mathrm{SOC}$, i.e. the ratio between the charge $Q_{\max }$ exiting (or entering) the cell during one single discharge (or charge) current peak and its nominal capacity $C_{\text {nom. }}$. More precisely:

$\Delta T_{\max }=T_{\max }-T_{\mathrm{amb}}$

$\Delta S O C_{\max }=\frac{Q_{\max }}{C_{\text {nom }}}=\frac{\int_{a}^{b} i(t) d t}{C_{\text {nom }}}$

Results show that temperature remain within limits, since the maximum admitted temperature for the considered cell is $60^{\circ} \mathrm{C}$. The stress, in case of charge currents of $10 C_{\mathrm{n}}$ maintained for $20 \mathrm{~s}$, must be considered very severe, since it tends to begin continuous, but exceeding more than three times the suggested value by manufacturer (i.e. $3 C_{\mathrm{n}}$ ). In terms of maximum $\mathrm{SOC}$ variation, when executed at maximum current rates $\left(\begin{array}{ll}10 & C_{\mathrm{n}}\end{array}\right)$ and durations very close to the typical hybrid vehicle usage (i.e. $10 \mathrm{~s}$ and $20 \mathrm{~s}$ ), it was respectively equal to $2.8 \%$ and $5.5 \%$ respectively. 
Also super high power cell was considered, on analogue test conditions. In particular, it was possible to observe that for reduced current amplitudes and pulse durations, i.e. up to $5 C_{\mathrm{n}}$, the maximum registered cell over-temperature was quite the same, i.e. around $4^{\circ} \mathrm{C}$. On the other hand, moving up to $10 C_{\mathrm{n}}$, the overtemperature strongly raised up in case of high power cell, around $15.5^{\circ} \mathrm{C}$ as visible from Table 3 . Instead, in case of super high power cell it remains significantly shorter. In fact, in the worst case that was considered, i.e. $10 C_{\mathrm{n}}$ and 30 seconds, the over-temperature is limited within $7^{\circ} \mathrm{C}$.

Global results of type B test, performed on the high power cell in a climatic chamber at $22^{\circ} \mathrm{C}$, are summarised in Table 4. A sample of type B test related to a peak amplitude of $10 C_{\mathrm{n}}$ and duration of $10 \mathrm{~s}$, is shown in Figure 6.

As visible, the linear variable current stress, at equal amplitude and duration, causes lower cell overtemperature and smaller SOC variation, since the average current is lower than in the equivalent A type test. In this case, the maximum SOC variation was respectively, depending on the considered duration (i.e. $10 \mathrm{~s}$ and $20 \mathrm{~s}$ ), equal to $1.4 \%$ and $2.8 \%$.

Finally, test of type $\mathrm{C}$ was performed on the super high power cell. The obtained result confirms also in this case a reduced over-temperature, as shown in Figure 7. As noted, in this type of test related to one real operating condition, the current was shaped in such a way that positive and negative peaks were equal, respectively to $6 C_{\mathrm{n}}$. It is clearly seen from these plots that this stress does not cause important overtemperature in the cell, even though peaks reach $6 C_{\mathrm{n}}$ not only during discharging conditions, but also during charging. In this case, SOC variations are not easily to evaluate as in the previous case, due the complexity of the considered profile. However, the maximum SOC variation was about $2.2 \%$ in the NEDC urban part, and about $3.5 \%$ in the NEDC extra-urban one.

As it is possible to summarise, in all the examined cases it is clearly seen that these stresses do not cause significant overheating, even though current peaks during charge are much more than what considered acceptable by the manufacturer. Indeed, it may be questioned if these current stresses could imply a short cycle life, as discussed in the next section. 


\section{Cycle life evaluation}

Experimental evaluation of cycle life has been performed having as reference the type B micro-cycle, in the form already shown in Figure 6 (i.e. $10 \mathrm{C}_{\mathrm{n}}, 10 \mathrm{~s}$ ), i.e. corresponding to a maximum SOC variation of $1.4 \%$, being much more realistic of usage in hybrid vehicles. The test was composed by the repetition of many micro-cycles with periodic check of the cell capacity. The test is arranged so that:

- Before start, the cell has been subjected to five training full depth charge-discharge cycles.

- Around 1200 micro-cycles per day have been executed (each micro-cycle has a total duration of $60 \mathrm{~s})$, typically divided in three sessions.

- Before starting each session, a constant voltage charge phase at $3.6 \mathrm{~V}$ is imposed, to compensate the charge deviation during micro-cycling. The correspondence between open circuit voltage and SOC is discussed in [36], [37], [38]. The value was chosen to not overcome the maximum and minimum allowable voltage, thus avoiding the "cut" already shown in the first part of Figure 5. In fact, at regime ( $\Delta \mathrm{T}$ constant) the window voltage must remain quite far from the upper and lower limits.

- Before starting the test, and after around every $10^{4}$ micro-cycles, a full depth charge-discharge cycle at $22^{\circ} \mathrm{C}$ is made, to verify the effective capacity of the cell. The capacity is measured integrating the charge exiting the cell during discharge, from $4.2 \mathrm{~V}$ up to $2.7 \mathrm{~V}$.

- Before starting the test, and also during each session, internal resistance has been directly evaluated from the measured voltage and current, according to the methodology followed also in [36] [38].

The full test duration was around nine months. After about $2 \cdot 10^{5}$ micro-cycles, the capacity was substantially unchanged respect to the initial value, nearly of $12.0 \mathrm{Ah}$, as shown in Table 5. Also internal resistance has suffered very small changes, as always visible from Table 5. Figure 8 shows the corresponding plots of capacity and internal resistance vs number of micro-cycles.

From the obtained results it is possible to conclude that this kind of stress has a very low impact on the cell life, mainly in comparison to full-depth cycles, although performed at lower current rates. Starting from these results, although no guarantee exists of a linear behavior of the cell, it appears reasonable to assume that the end of life could be effectively estimated in hundreds of thousands micro-cycles, according to the initial hypothesis. 
Among other, it remains also questionable whether under this kind of stress what value must be considered ad end-of-life capacity. For deep cycles it is typically $80 \%$ of its nominal value. In this case, since energy performance related to long discharge durations is not required, it appears reasonable to use as endof-life capacity a lower value, e.g. 50-60\% of the initial capacity.

As mentioned, the test duration was around nine months. During that period some interruptions occurred. Although very few unwanted events were registered, in a reduced number of cases they determined extrastresses for the cell, in particular discharging it under the imposed value, or maintaining the high charging current for more time than imposed. As example, the feeder did not stop the linear charging phase, remaining locked at high charging current values. Since the maximum voltage of the feeder was set slightly higher than the maximum cell voltage obtained during the test, failure in current regulation does not produce any overvoltage. Thus, voltage tends to remain confined to $4 \mathrm{~V}$, while current slowly decreases to zero, in a time of around $400 \mathrm{~s}$, limiting the stay at high currents for few tens of seconds. These events can be intended as an additional stress for the cell, that in any case it was substantially unaltered, as data of Table 5 have shown.

Before the test here described, the authors had experience of another experimental evaluation of cycle life, performed on super high power cell under test of type C. During this test, the cell was subjected to many reduced power NEDC cycles, also in this case with period check of cell capacity. The test, mostly detailed in [31], was arranged to perform a SOC balance, after each NEDC cycle, to avoid SOC deviation during repetition of cycles. Then, after 136 NEDC cycles, a complete charging-discharging cycle is imposed, to verify the capacity of the cell. Also in this case, reduction in terms of capacity was very limited, reduced to $1.8 \%$ after 1000 cycles, equivalent to around $10^{4} \mathrm{~km}$. Under the hypothesis to consider the ended capacity at $50 \%$ of its nominal value, it is possible to estimate by extrapolation around $3 \cdot 10^{5} \mathrm{~km}$, thus entirely covering the whole vehicle life.

This result, although not easily evaluable in terms of micro-cycles due the complexity of the current profile shown in Figure 4, tends to confirm the high vocation of high power cells in micro-cycling usage, as already noted in the extend cycle life above described. 


\section{Conclusions}

This paper has effectively shown that commercially available high power batteries are absolutely adequate to be used in "power oriented" applications, since:

- High charging or discharging currents up to $10 C_{\mathrm{n}}$, maintained for duration in order of seconds or tens of seconds, do not represent a significant thermal stress for the lithium cell.

- Cycle life evaluation, although actually in progress, has already shown that under the considered stress the cell is able to perform hundreds of thousands of micro-cycles, before showing a significant degradation.

- These results have strong impact also in regard to practical applications, since from the correlation between $\triangle \mathrm{SOC}$ and cycle life it is possible to better size the storage system, allowing a significant optimization of its mass, volume and cost. 


\section{References}

[1] A. Di Donato, G. Lutzemberger, D. Poli in: International Conference on Engines and Vehicles (ICE2009), Capri, 13-18 September 2009, http://dx.doi.org/10.4271/2009-24-0084.

[2] M. Ceraolo, N. Doveri, G. Lutzemberger in: International Symposium on Power Electronics, Electrical Drives, Automation and Motion (SPEEDAM), Pisa, 14-16 June 2010, http://dx.doi.org/10.1109/SPEEDAM.2010.5542074.

[3] T. Huria, G. Lutzemberger, G. Pede, G. Sanna in: IEEE Vehicle Power and Propulsion Conference (VPPC), 1-3 Sept. 2010, http://dx.doi.org/10.1109/VPPC.2010.5729243.

[4] M. Ceraolo, G. Lutzemberger, R. Mati, L. Sani in: Energy Conference and Exhibition (ENERGYCON), 9-12 Sept. 2012, http://dx.doi.org/10.1109/EnergyCon.2012.6347718.

[5] A. Bertini, M. Ceraolo, G. Lutzemberger, Automation in Construction 58 (2015) 144-154, http://dx.doi.org/10.1016/j.autcon.2015.07.013.

[6] M. Ceraolo, M. Conte, R. Giglioli, G. Lutzemberger, M. Pasquali in: AEIT Annual Conference, Oct. 2013, http://dx.doi.org/10.1109/AEIT.2013.6666810.

[7] M. Ceraolo, G. Lutzemberger, Journal of Power Sources 264 (2014) 128-139, http://dx.doi.org/10.1016/j.jpowsour.2014.04.070.

[8] S. Barsali, R. Giglioli, M. Giuntoli, G. Lutzemberger, D. Poli in: International Conference on Environment and Electrical Engineering (EEEIC), 10-13 Jun. 2015, http://dx.doi.org/10.1109/EEEIC.2015.7165546.

[9] Kokam documentation: http://www.kokam.com/english/product/battery_main.htm

[10] J. Guo, Z. Li, M. Pecht, Journal of Power Sources 281 (2015) 173-184.

[11] M. Broussely, S. Herreyre, P. Biensan et alii, Journal of Power Sources 146 (2005), 90-96.

[12] T. Wang, L. Pei et alii, Journal of Power Sources 301 (2016), 187-193. 
[13] K. Shah, S.J. Drake, D.A. Wetz, et alii, Journal of Power Sources 271 (2014) 262-268.

[14] M. Broussely, S. Herreyre, P. Biensan et alii, Journal of Power Sources 97/98 (2001), 13-21.

[15] S. Torai, M. Nakagomi, S. Yoshitake et alii, Journal of Power Sources 306 (2016), 62-69.

[16] I. J. Gordon, S. Genies, G. S. Larbi et alii, Journal of Power Sources 307 (2016), 788-795.

[17] B. Stiaszny, J.C. Ziegler, E. E. Krauss et alii, Journal of Power Sources 251 (2014), 439-450.

[18] B. Stiaszny, J.C. Ziegler, E. E. Krauss et alii, Journal of Power Sources 258 (2014), 61-75.

[19] M. Klett, R. Eriksson, J. Groot et alii, Journal of Power Sources 257 (2014), 126-137.

[20] G. Ning, B. Haran, B. Popov, Journal of Power Sources 117 (2003) 160-169.

[21] Y. Cui, C. Du, G. Yin, Y. Gao, et alii, Journal of Power Sources 279 (2015), 123-132.

[22] P.Van den Bossche, J. Van Mierlo, Applied Energy 113 (2014), 1575-1585.

[23] D. Wong, B. Shrestha, D. A. Wetz et alii, Journal of Power Sources 280 (2015), 363-372.

[24] E. Wood, M. Alexander, T.H. Bradley, Journal of Power Sources 196 (2011), 5147-5154.

[25] A. Marongiu, M. Roscher, D.U. Sauer, Applied Energy 137 (2015), 899-912.

[26] X. Han, M. Ouyang, L. Lu, Journal of Power Sources 251 (2014), 38-54.

[27] M. Grutzke, V. Kraft, B. Hoffmann et alii, Journal of Power Sources 273 (2015), 83-88.

[28] FreedomCAR Battery Test Manual: http://avt.inl.gov/battery/pdf/freedomcar_manual_04_15_03.pdf

[29] ISO 12405-2 Electrically propelled road vehicles - Test specification for lithium-ion traction battery packs and systems.

[30] G. Lutzemberger in: International Youth Conference on Energy (IYCE), 27-30 May 2015, http://dx.doi.org/10.1109/IYCE.2015.7180788. 
[31] M. Ceraolo, G. Lutzemberger, M. Marracci in: Vehicle Power and Propulsion Conference (VPPC), 1-3 Sept. 2010, http://dx.doi.org/10.1109/VPPC.2010.5729096.

[32] M. Ceraolo, S. Barsali, G. Lutzemberger, M. Marracci in: International Conference on Engines and Vehicles (ICE2009), Capri, 13-18 September 2009, http://dx.doi.org/10.4271/2009-24-0069.

[33] Maxwell technologies documentation: http://www.maxwell.com/products/ultracapacitors/

[34] Standardised driving cycles documentation: http://www.dieselnet.com/standards/cycles

[35] M. Ceraolo, A. di Donato, G. Franceschi, IEEE Transactions on Vehicular Technology 57 (2008) 14331441.

[36] M. Ceraolo, T. Huria, G. Lutzemberger in: SAE 2011 World Congress \& Exhibition, SAE Technical Paper, 2011-01-1365, http://dx.doi.org/10.4271/2011-01-1365.

[37] M. Ceraolo, T. Huria et alii in: SAE 2013 World Congress \& Exhibition, SAE Technical Paper, 201301-1547, http://dx.doi.org/10.4271/2013-01-1547.

[38] T. Huria, G. Ludovici, G. Lutzemberger, Journal of Power Source 249 (2014) 92-102, http://dx.doi.org/10.1016/j.jpowsour.2013.10.079. 
Table list

Table 1: Cell performance under constant current discharge, high power cell

Table 2: Main cell characteristics

Table 3: $\triangle \mathrm{SOC}$, cell over-temperature from ambient $\left(22^{\circ} \mathrm{C}\right)$ in type $\mathrm{A}$ test, high power cell

Table 4: $\triangle S O C$, cell over-temperature from ambient $\left(22^{\circ} \mathrm{C}\right)$ in type $\mathrm{B}$ test, high power cell

Table 5: Results of aging test, internal resistance and capacity check 
Figure captions

Figure 1. Ragone plot for the two considered devices

Figure 2. Thermal monitoring of lithium cell, $3 C_{\mathrm{n}}$ charging, $10 C_{\mathrm{n}}$ discharging on left, $20 C_{\mathrm{n}}$ discharging on right

Figure 3. Partial charge-discharge cycle, constant current (type A) on left, partial charge-discharge cycle, linearly variable current (type B) on right

Figure 4. NEDC cycle for low powered vehicles (above, only two urban parts shown) and corresponding battery current (type C)

Figure 5. Cell voltage (top), current and temperature (bottom), sample test of type A, high power cell Figure 6. Cell voltage (top), current and temperature (bottom), sample test of type B, high power cell

Figure 7. Cell current (top) and temperature (bottom) during a sample test of type $C$, super high power cell

Figure 8. Results of aging test, capacity (left) and internal resistance (right) vs micro-cycles 